\title{
Security Efficiency Maximization for Multi-UAV-Aided Network With Mobile Edge Computing
}

\author{
Guangchen Mu* \\ School of Electronic Information Engineering, Henan Institute of Technology, XinXiang, China
}

\section{OPEN ACCESS}

Edited by: Wei Wu,

Nanjing University of Posts and Telecommunications, China

Reviewed by: Yi Song,

Huaiyin Normal University, China

Pei Li,

Nanjing University of Posts and

Telecommunications, China Hongjiang Lei,

Chongqing University of Posts and

Telecommunications, China

*Correspondence:

Guangchen Mu

hngxymgc@126.com

Specialty section:

This article was submitted to Mobile and Ubiquitous Computing,

a section of the journal

Frontiers in Computer Science

Received: 07 April 2021

Accepted: 11 May 2021

Published: 04 June 2021

Citation:

Mu G (2021) Security Efficiency Maximization for Multi-UAV-Aided

Network With Mobile

Edge Computing.

Front. Comput. Sci. 3:691854. doi: 10.3389/fcomp.2021.691854
In this study, we consider a security efficiency maximization problem in a multiple unmanned aerial vehicle (UAV)-aided system with mobile edge computing (MEC). Two kinds of UAVs, including multiple computing UAVs (CUAVs) and multiple jamming UAVs (JUAVs), are considered in this system. CUAVs would receive partial computation bits and send the computation results to ground users. JUAVs do not undertake computing tasks and only send interference signals to counter potential ground eavesdroppers. We jointly optimize the ground user scheduling, UAV power, and UAV trajectory to maximize the security efficiency. The original problem is non-convex and difficult to solve. We first use the Dinkelbach method combined with continuous convex approximation technology, and then propose three corresponding subproblems, including user scheduling subproblem, UAV power subproblem, and UAV trajectory problem. Further, we apply the branch and bound method to solve the user scheduling subproblem, and optimize the two remaining subproblems by introducing auxiliary variables and Taylor expansion. The simulation results show that the proposed scheme can obtain better secure off-loading efficiency with respect to the existing schemes.

Keywords: mobile edge computing, multi-UAV, computation efficiency, secure transmission, jamming, trajectory scheduling

\section{INTRODUCTION}

The application of $5 \mathrm{G}$ mobile communication technology has brought many conveniences to people's life and production activities. The 5G network meets the needs of users for high bandwidth, low latency, and a large number of connected services. MEC is an important application in the $5 \mathrm{G}$ era. MEC provides local off-loading, which can effectively alleviate the data transmission pressure of the core network. In addition, mobile edge computing has flexible routing, efficient computing and storage capabilities, and can solve the key challenges of realizing the 5G vision (Pham et al., 2020). In many emerging applications, the demand for MEC is mainly reflected in the three aspects of delay, bandwidth, and security (Zhang et al., 2019). For instance, due to the limited battery capacity and energy consumption of mobile devices, MEC brings storage and computing resources to the edge of the network, enabling programs to run on mobile devices and meet latency requirements (Mach and Becvar, 2017). MEC-enabled loV can meet its heterogeneous needs for communication, computing, and storage, and to minimize the energy consumption of RSU (Ning et al., 2019). MEC cooperates with cloud computing to off-load the computing tasks in the vehicle network to the car, which can effectively reduce computing time and improve system practicability (Zhao 
et al., 2019). With the emergence of 5G, the industrial Internet is also developing rapidly. Data cleaning based on mobile edge nodes can maintain the reliability and integrity of data while improving the efficiency of data cleaning and reducing the energy consumption of industrial sensor cloud systems (Wang et al., 2020). In order to solve the problem of limited battery lifetime and low computing power of wireless devices, Mozaffari et al. (2019) proposed an MEC system supported by wireless power transfer to enhance computational capability. In the study by Han et al. (2020), the authors proposed a new algorithm to measure the efficiency of mobile edge computing systems. The maximum efficiency of MEC can be jointly optimized by the unloading time, CPU frequency, transmit power of users, and UAV trajectory (Bi and Zhang, 2018). Ding et al. (2019) studied the energy-saving resource allocation of MEC networks using NOMA.

MEC applications are deployed on general-purpose servers and are close to users, so UAVs are used to assist mobile edge computing. UAV-assisted MEC expands the coverage area of edge computing and improves computing capabilities. Due to the high mobility and flexibility of UAVs (Zeng and Zhang, 2017), UAV communication technology is widely used in many fields of electronic design, leading to rapid development of wireless communication technology (Gupta et al., 2016; Wu et al., 2018). Among them, Wu et al. (2018) considered a multi-UAV-enabled wireless communication system to provide services for ground users. UAVs can quickly cover the target area and divert services, which have a wide range of application scenarios (Sebastian and Jochen, 2014; Nader et al., 2018). For example, in smart cities, UAVs can be used for traffic management, environmental monitoring, pollution monitoring, and civil defense control (Hua et al., 2018). The use of drone aerial survey technology can effectively improve the efficiency and accuracy of topographic surveying and mapping work (Roberge et al., 2013). In the military field, UAVs can plan routes. Specifically, UAVs can plan a safe path in an obstacle environment according to their environment and maneuverability (Cao et al., 2018). When mobile edge computing is used to offload computing tasks to UAVs, the off-loaded information may be destroyed by eavesdroppers. With the increasing demand for global cellular network coverage, UAVs combined with cellular networks can support UAV communications in a low-cost and highly maneuverable manner (Wu et al., 2018). As an aerial base station, UAVs help the ground macro base station to provide high-rate data coverage when there are multiple or large service demands in the target area (Sun et al., 2019). In the cellular-connected UAV mobile edge computing system, parts of the tasks are migrated to the terrestrial base station for calculation, and the other part is calculated on the UAV (Hua et al., 2018). When the cellular system is attacked by jamming, the UAV can select a relay strategy for the mobile users in the cellular system (Lu et al., 2020).

In order to improve traffic efficiency, the UAV-assisted MEC system with three-layer integrated architecture is used to solve the dynamic optimization problem of energy perception (Zhang et al., 2018). UAV-assisted MEC systems need to meet the requirements of low energy consumption and high computing bits (Loke, 2015; Zhou Z. et al., 2018). In the study by Zhou F. et al. (2018), the problem of maximizing computational efficiency is studied by jointly optimizing the computational shunt and the UAV trajectory design in the UAV-assisted MEC network. The problem of maximizing the unloading efficiency of the multi-UAV-assisted MEC system is studied.

The study considers the calculation of bits and energy consumption (Zhang et al., 2020). In order to expand the coverage of edge computing, multiple UAVs can be used to assist MEC systems (Narang et al., 2017). But UAV-assisted MEC systems are at risk of eavesdropping. Zhao et al. (2019) proposed an energy-saving computing off-loading technology focusing on physical layer security. UAVs are used to send jamming signals to combat against eavesdroppers and improve the transmission security rate (Li et al., 2019).

The rest of the article is organized as follows: Section 2 introduces the multiple UAV group-assisted MEC system and formulates the joint optimization problem. In Section 3, the optimal solution is proposed, while Section 4 details numerical simulations. Finally, Section $\mathbf{5}$ provides concluding remarks.

\section{THE SYSTEM MODEL AND PROBLEM FORMULATION}

\subsection{The System Model}

As shown in Figure 1, we consider a multiple UAV group-assisted MEC system, which includes $F_{1}$ jamming UAVs, $F_{2}$ UAVs, $K$ eavesdroppers, and $M$ legal smart mobile devices. A multiple UAV group has enormous computing ability, and its task is to fly from the initial position to the final position specified on the ground $S M D$. Legitimate smart mobile devices can receive data from outside. Due to the limited computing performance of ground mobile devices, some data are off-loaded to UAV via wireless links for auxiliary calculations. But there will be eavesdroppers monitoring the eavesdropping data. For the security of data transmission, the jamming UAVs are added and the jamming data are sent to eavesdroppers, and the system operates within a certain flight period $T$. For ease of explanation, the flight period is divided into $N$ slots with the same slot length. The slot length $\tau=T / N$ is small enough to allow the UAVs to fly at a small distance and approximate the channel gain within each slot.

Taking into account the 3-D Cartesian coordinate system, every legitimate device $S M D_{m} \in \mathcal{M}$ and the eavesdropper $K$ scattered on the ground irregularly, and their horizontal coordinates are given by $u_{j}=\left(x_{i}, y_{j}\right), j \in M \cup K$. Assume that all JUAVs are flying at a constant altitude $h_{1}$ above the ground, while UAVs are flying at a constant altitude $h_{2}$ above the ground. 


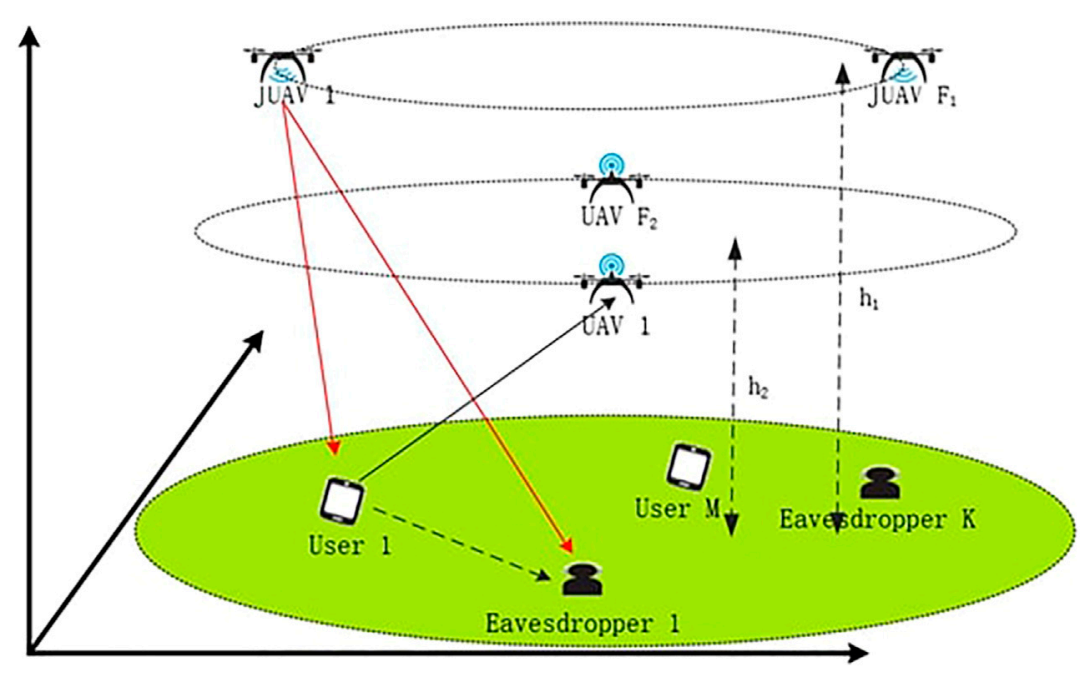

FIGURE 1 | ystem model.

The flight path of the slot projected by $J U A V_{i}, i \in F_{1}$ on the horizontal plane can be expressed as $q_{1, i}(n)=\left(x_{1, i}(n), y_{1, i}(n)\right)$. The flight path of slot $n$ projected by $U A V_{i}, i \in \mathcal{F}_{2}$ on the horizontal plane can be expressed as $q_{2, i}(n)=\left(x_{2, i}(n), y_{2, i}(n)\right)$.

Therefore, we can obtain the distance between UAVs $U A V_{i}, i \in \mathcal{F}_{1} \cup \mathcal{F}_{2}$ and any ground equipment $S M D_{j}, j \in \mathcal{M} \cup \mathcal{K}$ at a time slot according to the Euclidean formula, as follows:

$$
l_{i, j}(n)=\sqrt{h^{2}+\left\|q_{i}(n)-u_{j}\right\|^{2}}, h \in\left\{h_{1}, h_{2}\right\} .
$$

In general, for UAV-SMD wireless communication links, line-of-sight channels are more dominant than other channels. Similar to $\mathrm{Hu}$ et al. (2019) and Jeong et al. (2018), we consider that the wireless channel between ground equipment $S M D_{j}, j \in \mathcal{M} \cup \mathcal{K}$ and $\mathrm{UAV}$ $U A V_{i}, i \in \mathcal{F}_{1} \cup \mathcal{F}_{2}$ is a free space path loss model, which can be expressed as follows:

$$
h_{i, j}(n)=\beta_{0}\left(l_{i, j}(n)\right)^{-2},
$$

where $\beta_{0}$ is the channel power gain at the reference distance $l_{0}=$ $1 \mathrm{~m}$.

The channel from the legal device to the listener can be modeled as follows:

$$
g_{m, k}=\beta_{0}\left\|u_{i}\right\|^{-\varphi} \xi
$$

where $\varphi$ is the path loss index, $\xi$ is a random variable with exponential distribution, and its unit average represents smallscale Rayleigh fading.

For each legal device, its data can be calculated locally or its data can be off-loaded to the UAV for calculation. A binary variable $s_{m, f_{2}}(n) \in\{0,1\}$ is introduced to distinguish different states. The binary variable $s_{m, f_{2}}(n)=1$ indicates that the UAV provides services for SMD in the time slot $n$ and the two are associated, or it is 0 .

\subsubsection{Security Rate}

Here, a UAV can serve up to one legitimate user:

$$
\begin{aligned}
& \sum_{m=1}^{M} s_{m, f_{2}}[n] \leq 1, f_{2} \in \mathcal{F}_{2}, n \in \mathcal{N}, \\
& \sum_{f_{2}=1}^{F_{2}} s_{m, f_{2}}[n] \leq 1, m \in \mathcal{M}, n \in \mathcal{N}, \\
& s_{m, f_{2}} \in\{0,1\}, \forall m \in \mathcal{M}, f_{2} \in \mathcal{F}_{2}, n \in \mathcal{N} .
\end{aligned}
$$

Under the above conditions, the data transmission rate that the user can reach can be expressed as

$$
R_{m}[n]=\sum_{m=1}^{M} s_{m, f_{2}}[n] R_{m, f_{2}}[n],
$$

where

$$
R_{m, f_{2}}[n]=B \log _{2}\left(1+\frac{P_{m}[n] h_{m, f_{2}}[n]}{\sum_{i \in \mathcal{F}_{1}} P_{i}[n] h_{m, i}[n]+\sigma^{2}}\right),
$$

where $\sigma^{2}$ represents the power of the additive Gaussian white noise.

Similarly, the achievable rate of the $k$ th eavesdropper eavesdropping on user $m$ in time slots is given by

$$
R_{k \rightarrow m}[n]=\sum_{f_{2}=1}^{F_{2}} s_{m, f_{2}}[n] R_{m, k}[n],
$$

where

$$
R_{m, k}[n]=B \log _{2}\left(1+\frac{P_{m}[n] g_{m, k}[n]}{\sum_{i \in \mathcal{F}_{1}} P_{i}[n] h_{k, i}[n]+\sigma^{2}}\right) .
$$

Therefore, in the case of $k$ eavesdropper in the horizon time $T$, the worst-case security rate of the user $m \in \mathcal{M}$ is given by Zhu et al. (2017) and Zhu et al. (2016): 


$$
R_{m}^{\mathrm{sec}}=\sum_{n=1}^{N}\left(R_{m}[n]-\max _{k \in \mathcal{K}} R_{k \rightarrow m}[n]\right)^{+} .
$$

So the total expression is

$$
\begin{aligned}
R(X(n))= & R_{m}^{\mathrm{sec}} \\
= & \sum_{n=1}^{N} \sum_{m=1}^{M} s_{m, f_{2}}[n] B \log _{2}\left(1+\frac{P_{m}[n] h_{m, f_{2}}[n]}{\sum_{i \in \mathcal{F}_{1}} P_{i}[n] h_{m, i}[n]+\sigma^{2}}\right) \\
& -\max \sum_{k \in K_{f_{2}=1}} \sum^{F_{2}} B \log _{2}\left(1+\frac{P_{m}[n] g_{m, k}[n]}{\sum_{i \in \mathcal{F}_{1}} P_{i}[n] h_{k, i}[n]+\sigma^{2}}\right),
\end{aligned}
$$

in which $X[n]$ is the variable set of user association, system power, and trajectory of UAVs, that is, $X[n]=$ $\{s(n), p(n), q(n)\}$.

\subsubsection{System Energy}

The total energy consumption of the system consists of five parts: UAV transmission and unloading energy, UAV propulsion energy, ground equipment calculation energy, UAV calculation energy, and JUAV interference energy. According to Zeng and Zhang (2017), the propulsion energy of the UAV is much higher than other orders of magnitude, so only the propulsion energy of the UAV is calculated. The flight energy consumption of UAVs can be expressed as

$$
E_{i}=\sum_{n}^{N}\left(\left\|c_{1} v_{i}[n]\right\|^{3}+\frac{c_{2}}{\left\|v_{i}[n]\right\|}\left(1+\frac{\left\|a_{i}[n]\right\|^{2}}{g^{2}}\right)\right),
$$

where $g$ represents the acceleration of gravity, $c_{1}$ represents the wing area and air density constant of the $\mathrm{UAV}$, and $c_{2}$ represents the weight-dependent constant of the UAV.

As a result, the total expression is expressed as

$$
E(X(n))=\sum_{n}^{N}\left(\left\|c_{1} v_{i}[n]\right\|^{3}+\frac{c_{2}}{\left\|v_{i}[n]\right\|}\left(1+\frac{\left\|a_{i}[n]\right\|^{2}}{g^{2}}\right)\right) .
$$

According to Sun et al. (2019), the computational efficiency is considered to be the ratio of the total calculation bit to the total energy consumption, as given below:

$$
\eta=\frac{R(X(n))}{E(X(n))}
$$

\subsection{Problem Formulation}

This study focuses on maximizing the off-loading efficiency of multi-UAV-assisted edge computing, which jointly optimizes user resource scheduling, UAV flight power, andUAV trajectory. Based on the above analysis, the problem in this study can be expressed as

$$
\begin{aligned}
& P: \max _{X(n)} \eta \\
& C_{1}: h_{1} \neq h_{2} \\
& C_{2}: \sum_{m=1}^{M} s_{m, f_{2}}[n] \leq 1, f_{2} \in \mathcal{F}_{2}, n \in \mathcal{N}, s_{m, f_{2}} \in\{0,1\}, \\
& C_{3}: \sum_{f_{2}=1}^{F_{2}} s_{m, f_{2}}[n] \leq 1, m \in \mathcal{M}, n \in \mathcal{N}, s_{m, f_{2}} \in\{0,1\}, \\
& C_{4}: \sum_{f_{1}=1} s_{m, f_{2}}[n] \leq 1, k \in \mathcal{K}, n \in \mathcal{N}, s_{m, f_{1}} \in\{0,1\} \text {, } \\
& C_{5}: q_{i}[n+1]=q_{i}[n]+v_{i}[n] \tau+\frac{1}{2} a_{i}[n] \tau^{2}, n \in \mathcal{N}, i \in \mathcal{F}_{2}, \\
& C_{6}: v_{i}[n+1]=v_{i}[n]+a_{i}[n] \tau, n \in \mathcal{N}, i \in \mathcal{F}_{2}, \\
& C_{7}: q_{i}[0]=q_{i}[N+1], \quad i \in \mathcal{F}_{2} \text {, } \\
& C_{8}: v_{i}[0]=v_{i}[N+1], i \in \mathcal{F}_{2} \text {, } \\
& C_{9}:\left\|q_{i}[n+1]-q_{i}[n]\right\| \leq L^{2}, n \in \mathcal{N}, i \in \mathcal{F}_{2} \text {, } \\
& C_{10}:\left\|q_{j}[n]-q_{l}[n]\right\| \geq d^{2}, n \in \mathcal{N}, j, l \in \mathcal{F}_{2}, j \neq l, \\
& C_{11}: \sum_{n=1}^{N}\left\{\frac{f_{m}(n) \tau}{\chi}+\sum_{m=1}^{M} S_{m, f_{2}}[n] B \log _{2}\right. \\
& \left.\left(1+\frac{p_{m}[n] h_{m, f_{2}}[n]}{\sum_{i \in F_{1}} p_{i}[n] h_{m, i}[n]+\sigma^{2}}\right)\right\} \geq R_{\min } \\
& C_{12}: 0 \leq P_{i}[n] \leq P_{F_{\max }}, 0 \leq P_{m}[n] \leq P_{M_{\max }},
\end{aligned}
$$$$
i \in \mathcal{F}_{1} \cup \mathcal{F}_{2}, m \in \mathcal{M}, n \in \mathcal{N} \text {. }
$$

\section{PROPOSED SOLUTION}

In this section, the computational efficiency maximization problem is solved by the iterative optimization algorithm. Since problem $P$ is a nonlinear fractional problem, in order to make the problem easier to deal with, the Dinkelbach method is used to eliminate the fractional structure. Considering the coupling of user association, resource allocation, and trajectory scheduling, we decompose the original problem into three subproblems and propose an iterative optimization algorithm to solve it.

\subsection{Dinkelbach-Based Problem Reconstruction and Solution}

With the application of the Dinkelbach method $P$, solving a fractional programming problem transforms the original problem into a parameter programming problem on the basis of the $C_{1} C_{12}$ of the same constraint conditions. We introduce a parameter $\eta$ to represent the computational efficiency according to Dinkelbach (1967).

$$
\eta=\frac{R(X(n))}{E(X(n))}
$$

Assuming that $\eta^{*}$ is the optimal computational efficiency, the following Lemma can define the necessary and sufficient conditions for the optimal solution. 
Lemma 1: the optimal solution $X^{*}[n]$ can be obtained if and only if

$$
\max \left\{R(X(n))-\eta^{*} E(X(n))\right\}=0
$$

Proof: please refer to Dinkelbach (1967).

Problem $p$ can be transformed into a parameter problem $P 1$, according to Lemma 1, given as follows:

$$
\begin{gathered}
\max _{X[n]}\{R(X(n))-\eta E(X(n))\} \\
\text { s.t. } C_{1} C_{12},
\end{gathered}
$$

where $\eta$ is a nonnegative parameter. We then define the following equation:

$$
f(\eta)=\max _{X(n)}\{R(X(n))-\eta E(X(n))\},
$$

which is a strictly monotonic decreasing function for $\eta$ ( $\mathrm{Li}$ et al., 2019). If the optimal $\eta^{*}$ has been given in advance, we have $f(\eta) \geq 0$ for $\eta \leq \eta^{*}$; otherwise, we obtain $f(\eta)<0$ for $\eta>\eta^{*}$.

To obtain the optimal computational efficiency, a set of increments $\eta$ is obtained by the Dinkelbach method, and the value of $f(\eta)$ is approximately 0 by multiple iterations. The given $\eta$ can be obtained by studying $X(n)$ in problem $P_{1}$. Based on the correct $X(n)$, we then get the value of the new $\eta$. After several iterations, the optimal $\eta^{*}$ and $X^{*}(n)$ can be determined until $f\left(\eta^{*}\right)=0$ is satisfied.

\subsection{Joint Optimization}

Although problem $P_{1}$ has been relatively easy to handle, it is still non-convex due to the coupling between the variables of the UAV trajectory, user resource scheduling, and UAV power and spectrum resources. For decoupling non-convex problems, we decompose the problem $P_{1}$ into different subproblems and propose an iterative algorithm to solve them alternately.

\subsubsection{User Association Optimization}

We first propose the following definition:

$$
\stackrel{\wedge}{\stackrel{R}{R}\left[f_{2}\right.}[n]=R_{m, f_{2}}^{k}[n]+\nabla R_{m, f_{2}}^{k}[n]\left[\left\|t_{f_{2}}[n]-u_{m}\right\|-G^{k}[n]\right]+0,
$$

in which

$$
\begin{gathered}
R_{m, f_{2}}^{k}[n]=\sum_{n=1}^{N} \sum^{M} \sum^{F_{2}} S_{m, f_{2}}[n] B \log _{2} \\
\left(1+\frac{P_{m}[n]}{\left(\sum_{i \in F_{1}} P_{i}[n] l_{i, m}^{-2}+\delta^{2}\right)\left(h^{2}+\left\|t_{f_{x}}[n]-u_{m}\right\|^{2}\right)}\right) .
\end{gathered}
$$

According to the previous results, we formulate the following alternative problem:

$$
\begin{gathered}
\max \sum_{n=1}^{N} \sum_{m=1 f_{2}=1}^{M} \sum_{m, f_{2}}^{F_{2}}[n] \underset{m, f_{2}}{\stackrel{R}{R}}[n] \\
\text { s.t. }\left\|t_{i}(n)-t_{l}(n)\right\|^{2} \geq\left\|t_{i}^{k}[n]-t_{l}^{k}[n]\right\|^{2} \\
+2\left(t_{i}^{k}[n]-t_{l}^{k}[n]\right)^{T}\left(t_{i}[n]-t_{l}[n]\right) d_{\min }^{2} \leq \| t_{i}^{k}(n) \\
-t_{l}^{k}(n) \|^{2}+2\left(t_{i}^{k}(n)-t_{l}^{k}(n)\right)^{T}\left(t_{i}(n)-t_{l}(n)\right) \\
\sum_{n=1}^{N}\left(\frac{f_{m}(n) \tau}{X}+\sum_{m=1}^{M} S_{m, f_{2}}[n] \underset{m, f_{2}}{\stackrel{R}{X}[n])} \geq R_{\min } .\right.
\end{gathered}
$$

Equation 21 is a convex problem, which can be solved by the existing toolbox, such as CVX. We then focus on the resource allocation optimization.

\subsubsection{Resource Allocation Optimization}

In the case of given UAV user association and a flight trajectory, the power optimization of $\mathrm{P} 1$ in the problem can be changed into a new problem SP2 as follows.

$$
\begin{gathered}
\max _{P[n]} \sum_{m=1}^{M} S_{m, f_{2}[n]}\left[B \log _{2}\left(1+\frac{p_{m}[n] h_{m, f_{2}}[n]}{\sum_{i \in F_{1}} P_{i}[n] h_{m, f_{1}}[n]+\delta^{2}}\right)-B \log _{2}\right. \\
\left.\left(1+\frac{P_{m}[n] g_{m, k}[n]}{\sum_{i \in F_{1}} P_{i}[n] h_{k, f_{1}}[n]+\delta^{2}}\right)\right] \\
\text { s.t. } C_{11}, C_{12} .
\end{gathered}
$$

It can be noticed that power $p$ contains the UAV power pm and the jamming UAV power $P_{i}, i \in F_{1}$. The whole function is a non-convex function, and the two powers need to be discussed separately and transformed into convex functions.

$$
\alpha[n]=\frac{h_{m, f_{2}}[n]}{\sum_{i \in F_{1}} P_{i}[n] h_{m, f_{1}}[n]+\delta^{2}}, \beta[n]=\frac{g_{m, k}[n]}{\sum_{i \in F_{1}} P_{i}[n] h_{k, f_{1}}[n]+\delta^{2}} .
$$

It can be seen from the original formula of SP2 that $\alpha[n]>\beta[n]$. We then define a function as follows:

$$
f[n]=\frac{1+\alpha[n] P_{m}[n]}{1+\beta[n] P_{m}[n]},
$$

where $f[n]$ is a fraction, which can be transformed via the Dinkelbach method. Thus, we obtain the following version:

$$
\begin{aligned}
f[n] & =1+\alpha[n] P_{m}[n]-\lambda-\lambda \beta[n] P_{m}[n] \\
& =(1-\lambda)+(\alpha[n]-\lambda \beta[n]) P_{m}[n]
\end{aligned}
$$

As a result, we transform the original problem $p$ as follows:

$$
g_{p_{m}}[n]=\left\{B \log _{2}\left[(1-\lambda)+(\alpha[n]-\lambda \beta[n]) P_{m}[n]\right]\right\} \sum_{m=1}^{M} S_{m \cdot f_{2}}[n],
$$

whose gradient can be expressed as follows:

$$
\frac{\partial g[n]}{\partial P_{m}[n]}=\frac{(\alpha[n]-\lambda \beta[n]) \log _{2} e}{(1-\lambda)+(\alpha[n]-\lambda \beta[n]) P_{m}[n]} B \sum_{m=1}^{M} S_{m, f_{2}}[n] .
$$

We then carry out the first-order Taylor expansion of $g_{P_{m}}[n]$ as follows:

$$
g_{P_{m}}[n]=g_{P_{m}}\left[n_{0}\right]+\nabla g_{P_{m}}[n]\left(P_{m}[n]-P_{m}\left[n_{0}\right]\right) .
$$

Combining Eqs. 27, 28, we get the following problem:

$$
S P 2^{\prime}: \max _{P_{m}[n]} \sum_{m=1}^{M} S_{m, f_{2}[n]} \underset{p_{m}}{\hat{g}}[n] .
$$

Similarly, we transform the constraint $C_{11}$ as a convex form and obtain 


$$
\begin{aligned}
& S_{m, f_{2}}[n] B \log _{2}\left(1+\alpha[n] P_{m}[n]\right)-R_{\min } \leq 0 \\
& h_{P_{m}}[n]=\sum_{m=1}^{M} S_{m, f_{2}}[n] B \log _{2}\left(1+\alpha[n] P_{m}[n]\right) \\
& \frac{\partial h_{P_{m}}[n]}{\partial P_{m}[n]}=\frac{\alpha[n] \log _{2} e}{1+\alpha[n] P_{m}[n]} \sum_{m=1}^{M} S_{m, f_{2}}[n] B
\end{aligned}
$$

Further, we carry out the first-order Taylor expansion of $h_{P_{m}}[n]$ and transform it into the form of $\underset{P_{m}}{\hat{h}}[n]$. Finally, $C_{11}$ can be optimized to the following form:

$$
\sum_{n=1}^{N}\left(\frac{f_{m}(n) \tau}{X}+\sum_{m=1}^{M} S_{m, f_{x}} h_{p_{m}}[n]\right)>R_{\min } .
$$

The convex transformation of UAV power is discussed as above, and the following is the same for interfering with UAV power $P_{i}$.

$$
\begin{gathered}
S P 2: \max _{P[n]} B \log _{2}\left(1+\frac{p_{m}[n] h_{m, f_{2}}[n]}{\sum_{i \in F_{1}} P_{i}[n] h_{m, f_{1}}[n]+\delta^{2}}\right)-B \log _{2} \\
\left(1+\frac{P_{m}[n] g_{m, k}[n]}{\sum_{i \in F_{1}} P_{i}[n] h_{k, f_{1}}[n]+\delta^{2}}\right) .
\end{gathered}
$$

We get the definition as $v[n]=h_{m, f_{1}}[n], \mu[n]=h_{k, f_{1}}[n]$, $\gamma[n]=P_{m}[n] h_{m, f_{2}}[n]$, and $\varepsilon[n]=P_{m}[n] h_{m, k}[n]$. Then, the original problem is transformed into

$$
\max _{P_{i}[n]} B \log _{2}\left(\frac{1+\frac{\gamma[n]}{v[n] P_{i}[n]+\delta^{2}}}{1+\frac{\varepsilon[n]}{\mu[n] P_{i}[n]+\delta^{2}}}\right),
$$

which can be expressed as

$$
\max _{P_{i}[n]} B \log _{2}\left(\frac{\left(\mu[n] P_{i}[n]+\delta^{2}\right) \gamma[n]}{\left(\nu[n] P_{i}[n]+\delta^{2}\right) \varepsilon[n]}\right) .
$$

It can be seen that the original formula is not simple enough, so it should be further optimized. We assume $\mu[n] \gamma[n]=a[n]$, $\gamma[n] \delta^{2}=b[n], v[n] \varepsilon[n]=c[n]$, and $\delta^{2} \varepsilon[n]=d[n]$. Then the function $f[n]$ is transformed into

$$
f[n]=\frac{a[n] P_{i}[n]+b[n]}{c[n] P_{i}[n]+d[n]} .
$$

We similarly utilize the Dinkelbach method for the formula to optimize the fraction into an integral:

$$
g_{p_{i}}[n]=B \log _{2}\left[(a[n]-\lambda c[n]) P_{i}[n]+b[n]-\lambda d[n]\right],
$$

whose gradient can be expressed as

$$
\frac{\partial g_{p_{i}}[n]}{\partial P_{i}[n]}=\frac{(a[n]-\lambda c[n]) \log _{2} e}{\left[(\nu[n]-\lambda c[n]) P_{i}[n]+b[n]-\lambda d[n]\right]} B .
$$

According to the expression of $g_{P_{i}}^{\wedge}[n]$, we transform $S P 2$ into a new problem $S P 2^{\prime \prime}$ as follows:

$$
S P 2^{\prime \prime}: \max _{P_{i}[n]} S_{m, f_{x}[n]} \underset{p_{i}}{\hat{g}}[n] .
$$

\subsubsection{Trajectory Scheduling of UAVs}

Finally, we can optimize the trajectory scheduling of UAVs at a given user association and given power and frequency. The total
SMD energy consumption for processing computing tasks is as follows:

$$
\begin{aligned}
& E(X[n])=\sum_{n=1}^{N} \sum_{m=1}^{M} \sum_{k=1}^{K}\left\{\gamma f_{m}^{3}(n)+\sum_{f_{s}=1}^{F_{2}} S_{m, f_{2}}[n] P_{m, f_{2}}[n]\right. \\
& \left.+\sum_{f_{1}=1}^{F_{1}} S_{k, f_{1}}[n] P_{k, f_{1}}[n]\right\} \tau+\sum_{n=1}^{N}\left(a\left\|v_{i}[n]\right\|^{3}+\frac{c_{2}}{\left\|v_{i}[n]\right\|}\right. \\
& \left.\left(1+\frac{\left\|a_{i}[n]\right\|^{2}}{g^{2}}\right)\right)+\sum_{n=1}^{N} \sum_{k=1}^{K} r S_{m, f_{2}}[n] \rho_{k}[n] L_{k}[n] s_{k} c_{3}^{2},
\end{aligned}
$$

where the relaxation variable $\mu_{\mathrm{i}}[n]$ is used to replace the variable $\left\|v_{i}\right\|$ satisfying

$$
\begin{aligned}
\mu_{i}[n] & \geq 0, \\
\left\|v_{i}[n]\right\|^{2} & \geq \mu_{i}[n],
\end{aligned}
$$

which can be transformed into

$$
\left\|v_{i}[n]\right\|^{2} \geq\left\|v_{i}^{r}[n]\right\|^{2}+2\left(v_{i}^{r}[n]\right)^{T}\left(v_{i}[n]-v_{i}^{r}[n]\right) .
$$

It is worth noting that optimizing the original formula, we get the following formula:

$$
\sum \sum c_{1}\left\|v_{i}[n]\right\|^{3}+\frac{c_{2}}{\mu[n]}+\frac{c_{2}\left\|a_{i}[n]\right\|^{2}}{\mu_{i}[n] g^{2}} .
$$

In this way, the original problem becomes a convex expression as in Eq. 42, which can be solved by CVX.

\section{NUMERICAL RESULTS}

In this section, we provide a visual representation of our proposed scheme and comparison scheme. Since the space-to-ground channel is a LoS channel, we further assume that the ground signal obeys Rayleigh fading. We use MATLAB to generate artificial data, and in each setting, 1,000 experiments are used to take the average of the results. Specific parameter setting is provided in Table 1.

Figure 2 shows the UAVs' flight trajectory and the location of the ground user. The red discrete points represent the flight trajectory of the interference UAV, and the black discrete points represent the flight trajectory of the UAV. The blue dots represent ground users, and the green dots represent eavesdroppers. It can be seen from the figure that the flying speed of the drone will slow down when it is close to the user, and the flying speed will increase when it is far away from the user.

Figure 3 represents how energy efficiency varies with the number of calculated bits. We have provided the results of four schemes for comparison: 1) the scheme proposed in this article, 2) the scheme without JUAV, 3) the case without eavesdroppers, and 4) the scheme with ground jammers. According to Figure 3, as the number of calculation bits increases, the energy efficiency gradually decreases. Furthermore, our proposed scheme has better performance than other benchmark schemes.

In Figure 4, it shows the variation of energy consumption with the number of bits. Since our proposed scheme is to maximize 
TABLE 1 | Simulation parameters.

Parameter

The total system bandwidth

The total task completion time

Number of time slots

The channel power gain at a reference distance of $d_{0}=1 \mathrm{~m}$

The noise power

The fixed altitude of the UAV

The maximum available speed of the UAV

The initial and final position of the UAV

The position of the ground users

The position of the eavesdroppers

The effective switched capacitance of the UAV

Required CPU cycles per bit

Task input data size

$\begin{array}{cc}\text { Symbol } & \text { Value } \\ B & 30 \mathrm{MHz} \\ T & 100 \mathrm{~s} \\ N & 50 \\ \beta_{0} & -30 \mathrm{~dB} \\ N_{0} & -60 \mathrm{~dB} \\ h_{1}=h_{2} & 100 \mathrm{~m} \\ V_{\max } & 10 \mathrm{~m} / \mathrm{s} \\ \mathbf{q}_{\mathbf{1}}, \mathbf{q}_{\mathbf{F}} & (0,0),(200,0) \\ \mathbf{u}_{1}, \mathbf{u}_{2} & (50,75),(150,75) \\ \mathbf{e}_{1}, \mathbf{e}_{2} & (0,75),(200,75) \\ \kappa & 10^{-28} \\ C_{k} & 1,000 \mathrm{cycles} / \mathrm{bit} \\ I_{\min } & 200 \mathrm{Kbits}\end{array}$

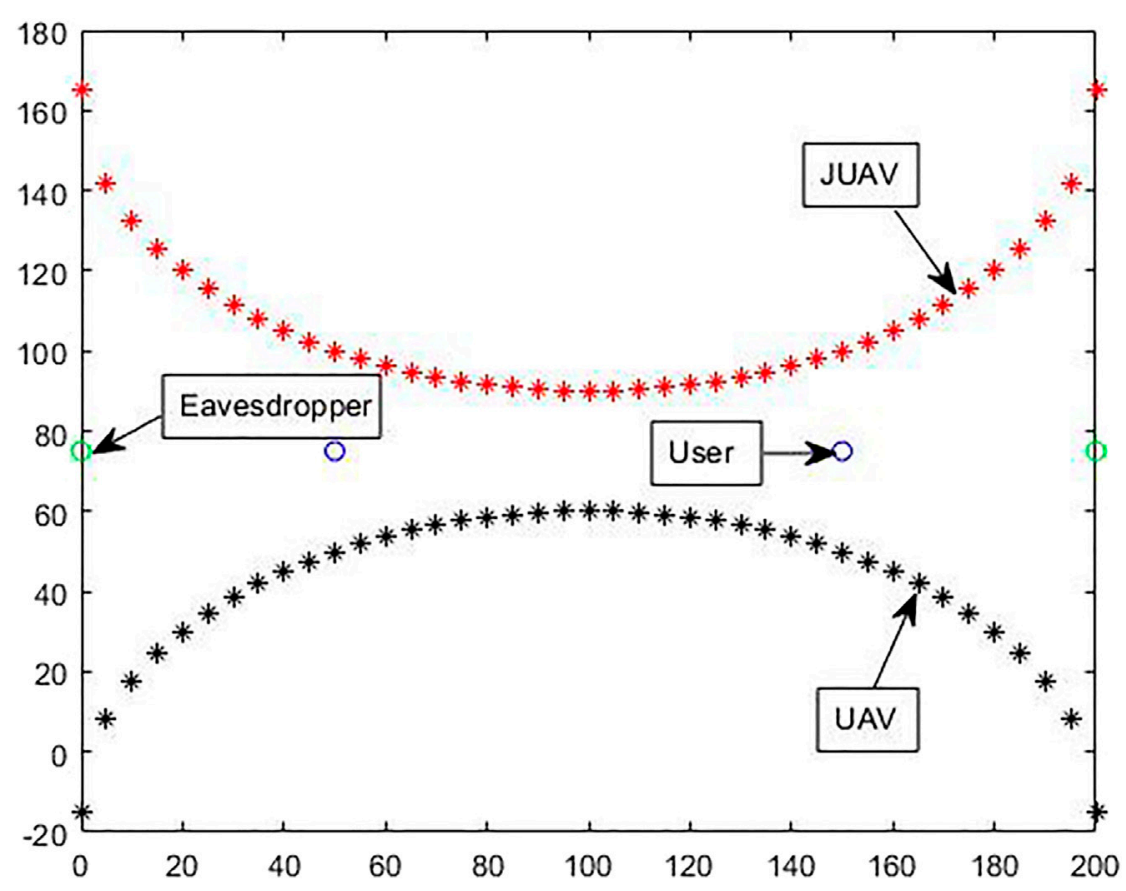

FIGURE 2 | UAVs' trajectory.

energy efficiency rather than minimizing energy consumption, we must consider whether the actual energy consumed is reasonable. The results in Figure 4 show that the proposed scheme still has better performance in terms of energy consumption.

In Figure 5, we show the energy efficiency curve with the maximum power on the drone. It is worth noting that as the maximum power of the UAV increases, the energy efficiency also increases. This is because when the energy is severely limited, the function of interfering with the eavesdropper and the auxiliary communication can seriously damage the safe rate. The greater the power of the UAVs, the less the impact of eavesdroppers.

\section{CONCLUSION AND DISCUSSION}

In this article, we propose a multi-UAVs-assisted mobile edge computing system in which different multiUAVs undertake computing tasks and interference tasks, respectively. We propose an alternate optimization algorithm and transform different subproblems into convex problems for easy solution. The simulation results prove the superiority of our proposed scheme.

It is worth noting that we assume that both the airto-ground channel and the terrestrial channel are perfectly known. However, in practical applications, the channel from the user to the potential eavesdropper is 


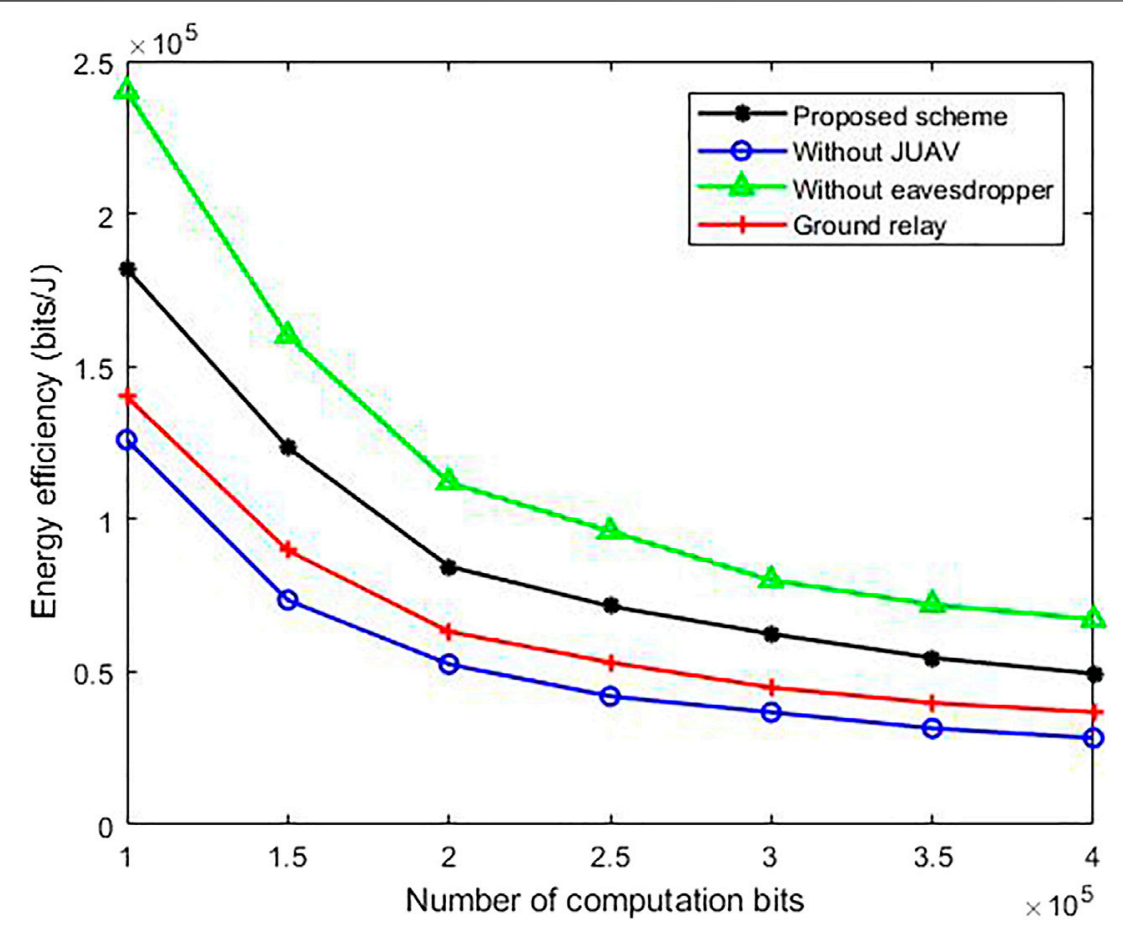

FIGURE 3 | Energy efficiency vs. the number of calculated bits.

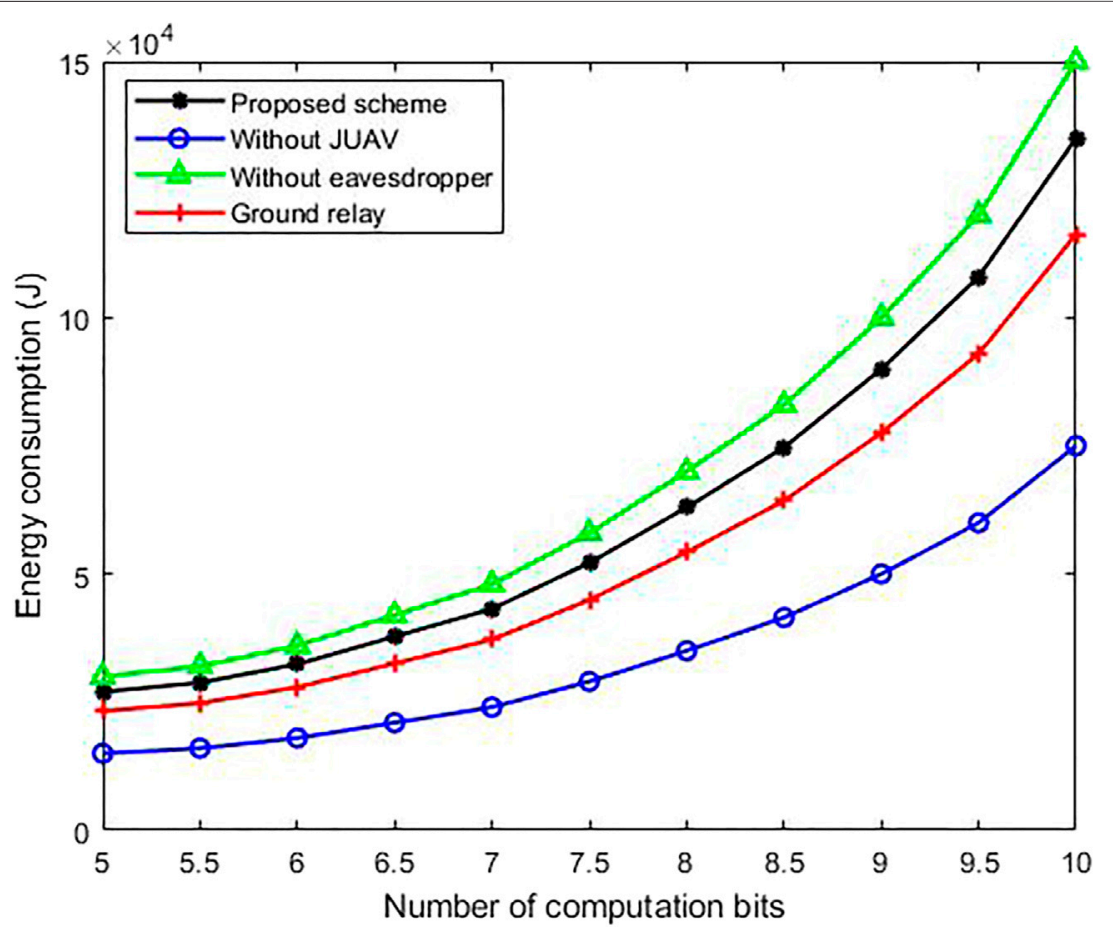

FIGURE 4 | Energy consumption vs. the number of calculated bits. 


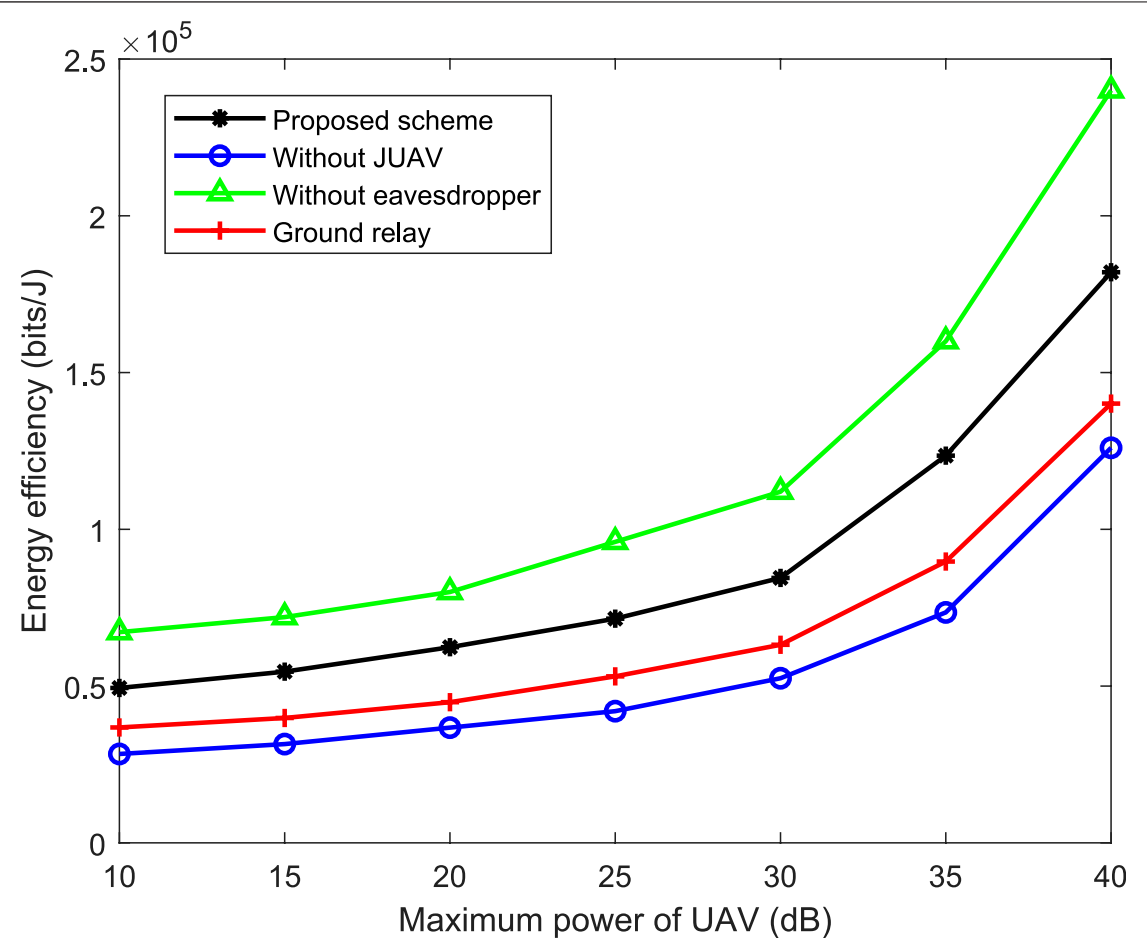

FIGURE 5 | Energy efficiency vs. the maximum power.

usually imperfect. In this case, how to enhance the robustness of the system is the direction of our follow-up work.

\section{DATA AVAILABILITY STATEMENT}

The original contributions presented in the study are included in the article/Supplementary Material; further inquiries can be directed to the corresponding author.

\section{REFERENCES}

Bi, S., and Zhang, Y. J. (2018). Computation Rate Maximization for Wireless Powered mobile-edge Computing with Binary Computation Offloading. IEEE Trans. Wireless Commun. 17, 4177-4190. doi:10.1109/TWC.2018.2821664

Cao, X., Xu, J., and Zhang, R. (2018). "Mobile Edge Computing for CellularConnected Uav: Computation Offloading and Trajectory Optimization," in 2018 IEEE 19th International Workshop on Signal Processing Advances in Wireless Communications (SPAWC), 1-5. doi:10.1109/SPAWC.2018.8445936

Ding, Z., Fan, P., and Poor, H. V. (2019). Impact of Non-orthogonal Multiple Access on the Offloading of mobile Edge Computing. IEEE Trans. Commun. 67, 375-390. doi:10.1109/TCOMM.2018.2870894

Dinkelbach, W. (1967). On Nonlinear Fractional Programming. Management Sci. 13. doi: $10.1287 / \mathrm{mnsc} \cdot 13.7 .492$

Gupta, L., Jain, R., and Vaszkun, G. (2016). Survey of Important Issues in Uav Communication Networks. IEEE Commun. Surv. Tutorials 18, 1123-1152. doi:10.1109/COMST.2015.2495297

Han, Q., Zheng, G., and Xu, C. (2020). "Energy-efficient Resource Allocation for mobile Edge Computing in Noma-Enabled Small Cell Networks," in 2020 IEEE

\section{AUTHOR CONTRIBUTION}

GM finished this paper independently, including the conception of the study, analysis and manuscript preparation, manuscript writing, and the experiment.

\section{FUNDING}

This work was supported in part by the key scientific and technological project of Henan Province, Grant 212102210558.

20th International Conference on Communication Technology (ICCT), 415-419. doi:10.1109/ICCT50939.2020.9295762

Hu, Q., Cai, Y., Yu, G., Qin, Z., Zhao, M., and Li, G. Y. (2019). Joint Offloading and Trajectory Design for Uav-Enabled mobile Edge Computing Systems. IEEE Internet Things J. 6, 1879-1892. doi:10.1109/JIOT.2018.2878876

Hua, M., Huang, Y., Wang, Y., Wu, Q., Dai, H., and Yang, L. (2018). Energy Optimization for Cellular-Connected Multi-Uav mobile Edge Computing Systems with Multi-Access Schemes. J. Commun. Inf. Networks 3 (4), 33-44. doi:10.1109/iccs.2018.8689226

Jeong, S., Simeone, O., and Kang, J. (2018). Mobile Edge Computing via a UavMounted Cloudlet: Optimization of Bit Allocation and Path Planning. IEEE Trans. Veh. Technol. 67, 2049-2063. doi:10.1109/TVT.2017.2706308

Li, A., Wu, Q., and Zhang, R. (2019). Uav-enabled Cooperative Jamming for Improving Secrecy of Ground Wiretap Channel. IEEE Wireless Commun. Lett. 8, 181-184. doi:10.1109/LWC.2018.2865774

Loke, S. W. (2015). The Internet of Flying-Things: Opportunities and Challenges with Airborne Fog Computing and mobile Cloud in the Clouds. Computer Sci.

Lu, X., Xiao, L., Dai, C., and Dai, H. (2020). Uav-aided Cellular Communications with Deep Reinforcement Learning against Jamming. IEEE Wireless Commun. 27, 48-53. doi:10.1109/MWC.001.1900207 
Mach, P., and Becvar, Z. (2017). Mobile Edge Computing: A Survey on Architecture and Computation Offloading. IEEE Commun. Surv. Tutorials 19, 1628-1656. doi:10.1109/COMST.2017.2682318

Mozaffari, M., Saad, W., Bennis, M., Nam, Y.-H., and Debbah, M. (2019). A Tutorial on Uavs for Wireless Networks: Applications, Challenges, and Open Problems. IEEE Commun. Surv. Tutorials 21, 2334-2360. doi:10.1109/COMST.2019.2902862

Nader, M., Jameela, A. J., Imad, J., Ahmed, I., and Farhan, M. (2018). Unmanned Aerial Vehicles Applications in Future Smart Cities. Technol. Forecast. Soc. Change 153, S0040162517314968. doi:10.1016/j.techfore.2018.05.004

Narang, M., Xiang, S., Liu, W., Gutierrez, J., Chiaraviglio, L., Sathiaseelan, A., et al. (2017). "Uav-assisted Edge Infrastructure for Challenged Networks," in 2017 IEEE Conference on Computer Communications Workshops, Atlanta, GA, United States, (INFOCOM WKSHPS), 60-65. doi:10.1109/INFCOMW.2017.8116353

Ning, Z., Huang, J., Wang, X., Rodrigues, J. J. P. C., and Guo, L. (2019). Mobile Edge Computing-Enabled Internet of Vehicles: Toward Energy-Efficient Scheduling. IEEE Netw. 33, 198-205. doi:10.1109/MNET.2019.1800309

Pham, Q.-V., Fang, F., Ha, V. N., Piran, M. J., Le, M., Le, L. B., et al. (2020). A Survey of Multi-Access Edge Computing in $5 \mathrm{~g}$ and beyond: Fundamentals, Technology Integration, and State-Of-The-Art. IEEE Access 8, 116974-117017. doi:10.1109/ACCESS.2020.3001277

Roberge, V., Tarbouchi, M., and Labonte, G. (2013). Comparison of Parallel Genetic Algorithm and Particle Swarm Optimization for Real-Time Uav Path Planning. IEEE Trans. Ind. Inf. 9, 132-141. doi:10.1109/TII.2012.2198665

Sebastian, S., and Jochen, T. (2014). Mobile 3d Mapping for Surveying Earthwork Projects Using an Unmanned Aerial Vehicle (Uav) System. SciencDirect Automation in Constructin. 41, 1-14. doi:10.1016/j.autcon.2014.01.004

Sun, H., Zhou, F., and Hu, R. Q. (2019). Joint Offloading and Computation Energy Efficiency Maximization in a mobile Edge Computing System. IEEE Trans. Veh. Technol. 68, 1. doi:10.1109/TVT.2019.2893094

Wang, T., Ke, H., Zheng, X., Wang, K., Sangaiah, A. K., and Liu, A. (2020). Big Data Cleaning Based on mobile Edge Computing in Industrial Sensor-Cloud. IEEE Trans. Ind. Inf. 16, 1321-1329. doi:10.1109/TII.2019.2938861

Wu, Q., Zeng, Y., and Zhang, R. (2018). Joint Trajectory and Communication Design for Multi-Uav Enabled Wireless Networks. IEEE Trans. Wireless Commun. 17, 2109-2121. doi:10.1109/TWC.2017.2789293

Zeng, Y., and Zhang, R. (2017). Energy-efficient Uav Communication with Trajectory Optimization. IEEE Trans. Wireless Commun. 16, 3747-3760. doi:10.1109/TWC.2017.2688328

Zhang, J., Zhou, L., Zhou, F., Seet, B.-C., Zhang, H., Cai, Z., et al. (2020). Computation-efficient Offloading and Trajectory Scheduling for Multi-Uav
Assisted mobile Edge Computing. IEEE Trans. Veh. Technol. 69, 2114-2125. doi:10.1109/TVT.2019.2960103

Zhang, L., Zhao, Z., Wu, Q., Zhao, H., Xu, H., and Wu, X. (2018). Energy-aware Dynamic Resource Allocation in Uav Assisted mobile Edge Computing over Social Internet of Vehicles. IEEE Access 6, 56700-56715. doi:10.1109/ACCESS. 2018.2872753

Zhang, X., Zhong, Y., Liu, P., Zhou, F., and Wang, Y. (2019). Resource Allocation for a Uav-Enabled mobile-edge Computing System: Computation Efficiency Maximization. IEEE Access 7, 113345-113354. doi:10.1109/ACCESS.2019. 2935217

Zhou, F., Wu, Y., Hu, R. Q., and Qian, Y. (2018). Computation Rate Maximization in Uav-Enabled Wireless-Powered mobile-edge Computing Systems. IEEE J. Select. Areas Commun. 36, 1927-1941. doi:10.1109/JSAC. 2018.2864426

Zhou, Z., Feng, J., Tan, L., He, Y., and Gong, J. (2018). An Air-Ground Integration Approach for mobile Edge Computing in Iot. IEEE Commun. Mag. 56, 40-47. doi:10.1109/MCOM.2018.1701111

Zhao, J., Li, Q., Gong, Y., and Zhang, K. (2019). Computation Offloading and Resource Allocation for Cloud Assisted mobile Edge Computing in Vehicular Networks. IEEE Trans. Veh. Technol. 68, 7944-7956. doi:10.1109/TVT.2019. 2917890

Zhu, Z., Chu, Z., Wang, N., Huang, S., Wang, Z., and Lee, I. (2017). Beamforming and Power Splitting Designs for An-Aided Secure Multi-User Mimo Swipt Systems. IEEE Trans.Inform.Forensic Secur. 12, 2861-2874. doi:10.1109/TIFS. 2017.2721908

Zhu, Z., Chu, Z., Wang, Z., and Lee, I. (2016). Outage Constrained Robust Beamforming for Secure Broadcasting Systems with Energy Harvesting. IEEE Trans. Wireless Commun. 15, 7610-7620. doi:10.1109/TWC.2016. 2605102

Conflict of Interest: The author declares that the research was conducted in the absence of any commercial or financial relationships that could be construed as a potential conflict of interest.

Copyright (c) $2021 \mathrm{Mu}$. This is an open-access article distributed under the terms of the Creative Commons Attribution License (CC BY). The use, distribution or reproduction in other forums is permitted, provided the original author(s) and the copyright owner(s) are credited and that the original publication in this journal is cited, in accordance with accepted academic practice. No use, distribution or reproduction is permitted which does not comply with these terms. 